\title{
Brinzolamide ophthalmic suspension: a review of its pharmacology and use in the treatment of open angle glaucoma and ocular hypertension
}

\author{
Michele lester \\ Clinica Oculistica, University \\ of Genoa, Italy
}

Correspondence: Michele lester Clinica Oculistica, University of Genoa, Viale Benedetto XV, I6I32 Genova, Italy Tel 0103537783

Fax 0103538494

Email iester@unige.it

\begin{abstract}
Brinzolamide is a white powder commercially formulated as a $1 \%$ ophthalmic suspension to reduce intraocular pressure (IOP). Pharmacologically, brinzolamide is a highly specific, non-competitive, reversible, and effective inhibitor of carbonic anhydrase II (CA-II), able to suppress formation of aqueous humor in the eye and thus to decrease IOP. Several clinical trials have evaluated its safety and the most commonly ocular adverse events are blurred vision (3\%-8\%), ocular discomfort (1.8\%-5.9\%), and eye pain $(0.7 \%-4.0 \%)$. Brinzolamide has been introduced to treat ocular hypertension and primary open-angle glaucoma. In some clinical studies it has been estimated that brinzolamide reduced IOP by was about $18 \%$. Brinzolamide can be added to beta-blockers and prostaglandins. In the latter combination, because prostaglandin derivatives improve the uveoscleral outflow but also increase the activity of CA in ciliary epithelium with a secondary increase in aqueous humor secretion, and slightly reduce the efficacy of prostaglandin analogues, theoretically topical CA inhibitors (CAI) decrease IOP by inhibiting CA-II, thus improving prostaglandin efficacy as well as lowering IOP. Brinzolamide could have a secondary possible effect on ocular flow too. Some clinical studies showed a mild improvement of ocular blood flow. Theoretically, CAI could give rise to metabolic acidosis, with secondary vasodilatation and improvement of blood flow. Systemic acidosis can occur in the setting of oral CAI therapy, and local acidosis within ocular tissues is theoretically possible with topical CAI therapy, with the potential for a local increase in ocular blood flow. In conclusion, topical CAI treatment has efficacy in IOP-lowering ranging from $15 \%$ to $20 \%$. From published data, brinzolamide can be used as first-line medication, even if other medications have a higher efficacy, with few side effects and it is a good adjunctive treatment. In some type of glaucoma patients with a vascular dysregulation, topical CAI could have a double effect: reducing IOP and improving ocular blood flow.
\end{abstract}

Keywords: brinzolamide, ocular hypertension, glaucoma, intraocular pressure, ocular blood flow, safety, treatment

Brinzolamide is a white powder insoluble in water, commercially formulated as a $1 \%$ ophthalmic suspension to reduce intraocular pressure (IOP). Brinzolamide empirical formula is $\mathrm{C}_{12} \mathrm{H}_{21} \mathrm{~N}_{3} \mathrm{O}_{5} \mathrm{~S}_{3}$ (Figure 1) with a melting point of approximately $131^{\circ} \mathrm{C}$ (Cvetkvic and Perry 2003; Shoji 2007). The commercially available preparation of brinzolamide is Azopt ${ }^{\circledR}$ (Alcon Laboratories, Inc, Ft. Worth, Texas, USA), with a $\mathrm{pH}$ of approximately 7.5 and osmolarity of $300 \mathrm{mOsm} / \mathrm{kg}$ (Cvetkvic and Perry 2003; Shoji 2007; Iester 2008).

The efficacy of brinzolamide $0.3 \%-3 \%$ bid has been evaluated in several randomized double-blind, multicenter comparative clinical trials (March and Ochsner 2000; Sall 2000; Shin 2000, 2000; Silver 1998, 2000; Michaud and Friren 2001). A dose-response study comparing brinzolamide in concentrations of $0.3 \%, 1 \%, 2 \%$, and 


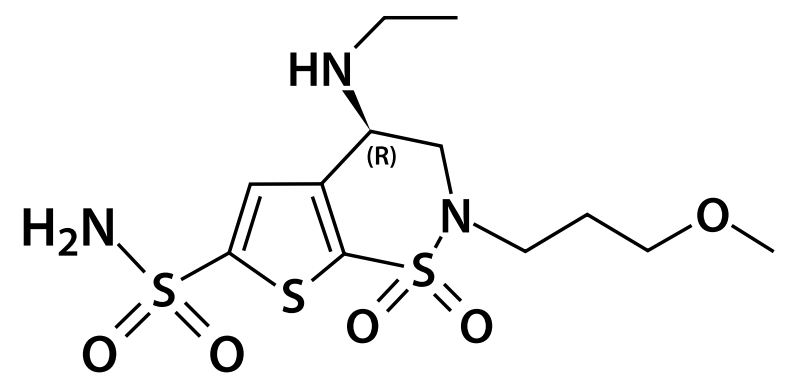

Figure I Chemical structure of brinzolamide.

$3 \%$ demonstrated mean IOP reductions of $3 \mathrm{mmHg}(11.3 \%)$, $4.3 \mathrm{mmHg}(16.1 \%), 4.4 \mathrm{mmHg}(16.1 \%)$, and $4.2 \mathrm{mmHg}$ (15.4\%), respectively. When diurnal IOP was measured, brinzolamide $1 \%$ or $3 \%$ reduced IOP significantly better than brinzolamide $0.3 \%$ (Silver 2000).

These observations suggested that the optimal therapeutic concentration for IOP reduction was $1 \%$. Its recommended dosing frequency is 3 times daily in the US and twice daily in the EU and Japan. However, 3 phase III trials have reported that brinzolamide $1 \%$ bid and tid produced statistically significant IOP reductions from baseline, and that both treatments were clinically equivalent to one another (Silver 1998; March and Ochsner 2000; Shin 2000).

Pharmacologically, brinzolamide is a highly specific, noncompetitive, reversible, and effective inhibitor of carbonic anhydrase II (CA-II) (DeSantis 2000), able to suppress formation of aqueous humor in the eye and thus decrease IOP. It can catalyze the reversible reaction of water and carbon dioxide $\left(\mathrm{CO}_{2}\right)$ to form negatively charged bicarbonate ions (Maren 1967). To achieve pharmacological effects, the near total inhibition of CA is required (Maren 1967; Kaur et al 2002). Brinzolamide is cleared from the aqueous humor and cornea with half-lives of approximately 3 and 5 hours respectively. Therefore, the cornea acts as a reservoir, providing sustained release of the drug to the ciliary processes of the ciliary body long after topical dosing (Maren 1967; Kaur et al 2002). In human tissues there are 7 different isoenzymatic forms. The most active isoenzymatic form is CA-II, which is found mainly in the erythrocytes, followed by kidneys, pancreas, eyes, central nervous system, and lungs (Maren 1967). In the eye, the enzyme is expressed mainly in the ciliary processes of the ciliary body, the corneal endothelial cells, and the pigment epithelium.

In the corneal endothelium CA-II plays a role in the pumping mechanism, which helps to maintain the relatively dehydrated state of the corneal stroma. Inhibition of this mechanism may lead to the development of corneal decompensation and edema, with secondary impaired vision.
In a randomized, double-blind clinical trial, 372 glaucomatous and ocular hypertension $(\mathrm{OH})$ patients received brinzolamide $1 \%$ or timolol $0.5 \%$. After 18 months of treatment, no significant change was found in corneal thickness and corneal endothelium cell density (March and Ochsner 2000). However, in this study only subjects with healthy corneas were included. Some concern remained in patients with compromised corneas, because in 2 published case reports corneal decompensation has been described in patients with keratopathy after treatment with dorzolamide (Adamson 1999; Konowal et al 1999).

After topical ocular administration systemic absorption of brinzolamide has been demonstrated in the tissues of healthy volunteers (DeSantis 2000). Topically instilled brinzolamide enters the blood circulation binding preferentially to CA in the erythrocytes leaving the concentration of free brinzolamide in plasma below the quantification level. In the red cells less than $1 \%$ of CA-II activity is required to maintain physiological function (March and Ochsner 2000). Thus in the erythrocytes the concentration of CA inhibitors (CAI) was insufficient to produce complete saturation of CA. In the kidney, free brinzolamide is not sufficient to inhibit in the proximal convoluted tubules and luminal CA; therefore systemic metabolic acidosis should not be obtained (see below). Furthermore, the low affinity of brinzolamide for the other CA isoforms and the low blood concentration may explain the low incidence of systemic adverse effects after topical administration (March and Ochsner 2000).

Several clinical trials (Zeyen and Caprioli 1993; Silver 1998; Sall 2000; Shin 2000; Wang et al 2004; Zhao and Chen 2005; Menon and Vernon 2006) have evaluated the safety of brinzolamide $1 \%$ ophthalmic suspension. The most commonly ocular adverse events were blurred vision $(3 \%-8 \%)$, ocular discomfort (1.8\%-5.9\%), and eye pain $(0.7 \%-4.0 \%)$. Other ocular adverse events occurring at an incidence of less than 3\% included hyperemia, pruritus, tearing, discharge, blepharitis, keratitis, foreign body sensation, dry eye, conjunctivitis, and lid margin crusting.

Zhau and Chen (2005) have reported two cases of corneal decompensation after 15 months and 2 years of brinzolamide therapy. Corneal edema reversed with discontinuation of the treatment; however, the reason was difficult to assess, and neither patient was rechallenged after recovery. No change in central corneal thickness was found (Wang et al 2004).

The most common systemic adverse event was taste perversion, occurring in 3.0\%-7.8\% of patients (Silver 1998; Sall 2000; Shin 2000). There were no clinically significant changes from baseline in heart rate and blood pressure, 
and in laboratory values for hematology, blood chemistry, or urinalysis variables. In addition, mean total carbonic anhydrase activity in red blood cells was reduced by only 51\%-55\%. However, one case of systemic metabolic acidosis has been described by Menon and Vernon (2006).

Brinzolamide has been introduced to treat $\mathrm{OH}$ and primary open-angle glaucoma (POAG), which are probably two different aspects of the same disease in which IOP is too high for the optic nerve head (ONH) structure.

Glaucoma is a multifactorial optic neuropathy characterized by the progressive loss of retinal ganglion cells and their axons. Clinically, the glaucomatous optic nerve manifests progressive enlargement of the optic cup, with commensurate progressive thinning of the neuroretinal rim and visual field defects. However, it is possible to lose up to $35 \%$ of optic nerve axons before the first visual field defect is detectable using standard automated perimetry (Pederson and Anderson 1980; Zeyen and Caprioli 1993).

$\mathrm{OH}$ is characterized by an increase of IOP without any visual field, $\mathrm{ONH}$, and retinal nerve fibre layer changes (European Glaucoma Society 2003). In other words $\mathrm{OH}$ can be a first step forward the glaucoma in most patients, but not all $\mathrm{OH}$ patients will change to glaucoma. Indeed, in the ocular hypertension treatment study (OHTS), it was found that after a follow-up of 5 or 7 years some $\mathrm{OH}$ subjects changed to glaucoma even if patients were treated or not (Van der Walk et al 2005). Obviously the percentage was different and in particular among the $\mathrm{OH}$ patients treated with topical hypotonic medication about $5 \%$ changed to glaucoma, while in the other group which did not receive any medication the percentage change was $9 \%$ (Kass et al 2002). However, it is still not clear when and which $\mathrm{OH}$ patients need to be treated.

Both in POAG and $\mathrm{OH}$ patients, the main risk factor for progression is an elevated IOP which can cause damage to the $\mathrm{ONH}$ and then to the visual field. Some clinical trials have shown that decreasing the IOP can be useful for slowing down glaucoma progression (CNTGS 1998; AGIS Investigators 2000; Kass et al 2001; Heijl et al 2002; Leske et al 2007), and IOP has been the only treatable risk factor until now (European Glaucoma Society 2003). Thus the treatment of glaucoma focuses on the reduction of IOP with drugs, lasers, or surgery. Over the past few years, there has been a gradual shift in the choice of first-line medical therapy. Six different classes of IOP-lowering molecules are currently available, including alpha adrenergic agonists, beta-blockers, CAI, parasympathomimetics, prostaglandin analogues, and sympathomimetics. The efficacy differs among these classes, ranging from about 30\% for the prostaglandins/ prostamides to about 18\% for the topical CAI (European Glaucoma Society 2003).

Clinically, all types of IOP-lowering medications can be used as the first choice, as European Glaucoma Society guidelines suggest. If the first-chosen medication alone does not control glaucoma, adjunctive therapy in the form of other topical agents can be added to the therapeutic regimen. However, when the first choice is not effective and/or tolerated in any individual patient, any of the other topical agents can be substituted as monotherapy.

When choosing the medical treatment for glaucoma, it is important to understand not only the aims of therapy, but also the mode of action, side effects, and contraindications of each individual medication (European Glaucoma Society 2003). Many antiglaucoma drugs are available, and the choice of therapy must take into account quality of life, cost, and compliance. If more than two topical medications are required to control the IOP, other forms of therapy, such as laser trabeculoplasty, or surgery, should be considered.

The reduction of IOP can differ based on the IOP baseline; however, the CNTGS showed that the reduction should be at least $30 \%$ to slow glaucoma progression (CNTGS 1998). In contrast the AGIS Investigators showed that in the group of patients that maintained IOP at around $12 \mathrm{mmHg}$ in all examinations during the 7-year follow-up, visual field did not progress (Reis et al 2006). Leske et al (2007) evaluated separately patients with higher and lower baseline IOP to determine progression factors at the end of the Early Manifest Glaucoma Trial (EMGT) based on all EMGT patients. Patients with early open-angle glaucoma were randomized to argon laser trabeculoplasty plus betaxolol or no immediate treatment, and examined every 3 months for up to 11 years. Treatment and follow-up continued to have a marked influence on IOP progression, regardless of baseline IOP. Other significant factors were age, bilaterality, exfoliation, and disc hemorrhages. Lower systolic perfusion pressure, lower systolic blood pressure, and cardiovascular disease history emerged as new predictors, suggesting a vascular role in glaucoma progression. A new risk factor was a thinner central corneal thickness (Leske et al 2007), but its role is still under debate as a confounding factor.

Also the OHTS showed that topical ocular hypotensive medication is effective in delaying or preventing the onset of POAG in individuals with elevated IOP $(\mathrm{OH})$ and no evidence of glaucomatous damage (European Glaucoma Society 2003). 
These data show that IOP reduction is fundamental in POAG and $\mathrm{OH}$ to delay the onset or prevent the onset of $\mathrm{POAG}$ in $\mathrm{OH}$ or the progression of the damage in POAG. Brinzolamide can be used alone or in combination with other medication to treat these patients.

The main ocular effect of brinzolamide is on IOP: in the ciliary epithelium, the inhibition of CA-II slows the formation of bicarbonate ions and their secretion into the posterior chamber of the eye. This reduces the sodium transport into the posterior chamber and decreases aqueous humor production, resulting in lower IOP (Maren 1967). Interestingly, CA is a highly efficient enzyme, and it has been estimated that more than $99 \%$ of CA activity must be inhibited in order to suppress aqueous production to lower IOP measurably (Maren 1967). The efficacy of brinzolamide $1 \%$ and dorzolamide $2 \%$ was compared in 25 normal subjects: brinzolamide reduced daytime aqueous flow by $0.47 \mu \mathrm{L} / \mathrm{min}$ and night-time aqueous flow by $0.16 \mu \mathrm{L} / \mathrm{min}$, while dorzolamide reduced aqueous flow by $0.34 \mu \mathrm{L} / \mathrm{min}$ and by $0.10 \mu \mathrm{L} / \mathrm{min}$, respectively. However, the relative reductions of aqueous flow achieved by brinzolamide and dorzolamide were similar for both medications (Ingram and Brubaker 1999).

\section{In monotherapy}

In a meta-analysis, Van der Valk et al (2005) estimated the IOP reduction achieved by the most frequently prescribed glaucoma drugs and a placebo in a meta-analysis of randomized clinical trials: they found that the highest reduction of IOP achieved by brinzolamide was of $17 \%$.

Wang et al (2004) conducted a small, prospective, double-masked study comparing brinzolamide $1 \%$ bid with timolol $0.5 \%$ bid in 50 patients with open-angle glaucoma. After 6 weeks of treatment, IOP dropped $4.8 \mathrm{mmHg}(17 \%)$ in brinzolamide-treated eyes and $5.7 \mathrm{mmHg}(19.7 \%)$ in timolol-treated eyes ( $p>0.05)$.

\section{Adjunctive to beta-blocker}

Shin (2000) compared the efficacy of brinzolamide 1\% (tid) and timolol $0.5 \%$ (bid) to timolol 0.5 (bid) and placebo (tid) in patients with open-angle glaucoma or $\mathrm{OH}$. After 1 month of timolol, patients added either brinzolamide or placebo for 3 months; mean IOP reductions after the addition of brinzolamide to timolol ranged from 3.3 to $4.1 \mathrm{mmHg}$ (statistically significant compared with both baseline and placebo).

\section{Adjunctive to prostaglandin}

As we well know, prostaglandin derivatives improve uveoscleral outflow, but they also increase the activity of CA in ciliary epithelium with a secondary increase in aqueous humor secretion (Martinez-de-la-Casa et al 2004), causing a slight reduction in the efficacy of prostaglandin analogues. Theoretically, topical CAI decrease IOP by inhibiting CA-II (DeSantis 2000), thus improving prostaglandin efficacy as well as reducing IOP.

Clinically in all adjunctive studies, a significant reduction of IOP was observed. Shoji et al (2005) added brinzolamide to once-daily latanoprost $0.005 \%$ in a small $(n=14)$, uncontrolled, open-label study of POAG. Mean IOP significantly ( $p<0.01$ ) decreased by $20 \%, 21.1 \%$, and $23.5 \%$ after 1,2 , and 3 months of treatment, respectively. Also in patients with normal-tension glaucoma, Nakamoto and Yasuda (2007) compared the efficacy of latanoprost alone in one eye with latanoprost plus brinzolamide in the fellow eye. Mean diurnal $(p<0.001)$ and nocturnal $(p<0.05)$ IOP and the mean circadian IOP reduction from washout $(p<0.01)$ were significantly reduced more in the brinzolamide adjunct group (17.1\%) than the latanoprost monotherapy group (13.6\%).

In a multicenter, randomized, double-masked, parallel group comparison study, Franks et al (2006) compared the effect of brinzolamide $1 \%$ bid or timolol $0.5 \%$ bid when added to travoprost. After 3 months, no significant $(p=0.96)$ difference in the IOP reduction was found between groups. Hollo et al (2006) found that patients receiving combined travoprost ophthalmic solution $0.004 \%$ and brinzolamide ophthalmic suspension 1\% therapy had lower IOP values than those on travoprost monotherapy $(p<0.0001)$. Combined therapy resulted in a significantly greater percentage of patients achieving IOP of $18 \mathrm{mmHg}$ or less $(\mathrm{p}<0.0001)$.

Feldman et al (2007) compared the efficacy of brinzolamide $1 \%$ bid with brimonidine $0.15 \%$ bid when added to travoprost once daily: after 3 months of treatment, a reduction of $2.7 \mathrm{mmHg}$ was found in the brinzolamide group and of $2.1 \mathrm{mmHg}$ in the brimonidine group (significant, $\mathrm{p}=0.035$ ).

Reis et al (2006) compared the additive efficacies of brinzolamide $1 \%$, brimonidine $0.2 \%$, or timolol $0.5 \%$, each dosed twice daily, when added to travoprost $0.004 \%$ once daily. Mean adjunctive IOP reduction seen with brinzolamide $(4.2 \mathrm{mmHg}, 22.7 \%)$ was statistically equivalent to that seen with timolol $(3.9 \mathrm{mmHg}, 20.2 \%)(\mathrm{p}>0.05)$ and both were significantly $(\mathrm{p}<0.02)$ greater than that seen with brimonidine (2.3 $\mathrm{mmHg}, 13.4 \%$ ).

Martinez-de-la-Casa et al (2004) compared the IOPlowering efficacy of concomitant therapy with travoprost once daily and brinzolamide twice daily versus once-daily dosing of the latanoprost/timolol fixed combination. Mean 
diurnal IOP was lower in the travoprost plus brinzolamide group than in the latanoprost/timolol group at 1 month $(p=0.041), 2$ months $(p=0.046)$, and 3 months $(p=0.015)$. The IOP differences ranged from 0.8 to $1.2 \mathrm{mmHg}$.

However, there is some published evidence that the pathophysiology of glaucoma is multifactorial, and that impaired blood flow has a role in the disease process (Leske et al 1995; Tielsch et al 1995; Bonomi et al 2000; Quigley et al 2001). Low diastolic perfusion pressure has been linked to a higher incidence of glaucoma in a population-based study. Furthermore, disc hemorrhage has been identified as a risk factor for disease progression in several studies (Budenz et al 2006; Leske et al 2003). Also, conditions related to vasospasm are sometimes found in patients with glaucoma, especially in normal-tension glaucoma. Vascular dysregulation could be one of the most important prognostic factors for following some patients. The inconstant $\mathrm{ONH}$ perfusion could activate the astroglia to produce some molecules that normally they do not produce or produce in low levels (Grieshaber et al 2007).

As Schulzer and Drance (1987) showed, the use of the magic number of $21 \mathrm{mmHg}$ to divide normal-tension glaucoma (NTG) from high-tension glaucoma (HTG) is not sufficient: indeed they found that other risk factors could be useful to divide POAG subgroups. Similar results using a different approach were obtained by Nicolela and Drance (1996) who reviewed about $1000 \mathrm{ONH}$ slides of glaucomatous patients to divide them into 4 glaucomatous subgroups: focal ischemic glaucoma, senile glaucoma, myopic glaucoma, and concentric excavation glaucoma. They selected about 100 pure aspect optic discs that were included in one of these four subgroups. Finally, they checked the clinical features of each subgroup and found significant differences in IOP values, vasospatic symptoms, and visual field defects, suggesting that IOP was still an important risk factor to treat, but other factors were involved in the pathogenesis of the disease.

Brinzolamide could have a secondary possible effect on ocular flow. Sampaolesi et al (2001) compared the ONH circulation in healthy eyes, $\mathrm{OH}$, and glaucoma eyes and found that blood flow was decreased in $\mathrm{OH}$ and glaucoma eyes compared with normal ones. Brinzolamide restored ONH blood flow to normal levels in $\mathrm{OH}$ eyes, but it did not change with brinzolamide therapy, most probably due to the loss of connective tissue.

However, Kothy and Hollo (2001) investigated the potential in vivo influence of different topical glaucoma medications on the diameter of retinal arterioles of healthy volunteers and glaucoma patients through the use of a retinal vessel analyzer (RVA, Imedos $\mathrm{GmbH}$, Weimar, Germany) and found no significant changes in arteriole diameter at 2 hours after instillation of the topical antiglaucoma drugs (ie, brinzolamide $1 \%$, timolol $0.5 \%$, betaxolol $0.5 \%$, brimonidine $0.2 \%$, latanoprost $0.005 \%$ ).

Siesky et al (2006) reported that eyes with POAG, treated with brinzolamide, benefited from higher diastolic perfusion pressures than eyes treated with dorzolamide. Thirteen subjects were enrolled in a masked crossover study consisting of 3 months of therapy with each drug. There were no statistically significant differences in IOP, heart rate, mean arterial pressure, or systolic blood pressure, but diastolic perfusion pressure was higher in left eyes (although comparable in right eyes) of subjects treated with brinzolamide versus dorzolamide.

Using the Heidelberg Retina Flowmeter in a prospective study on 20 glaucomatous patients, Iester (2003) found that after brinzolamide treatment a 19\% decrease in IOP was obtained and a concomitant improvement of the retinal blood flow was obtained at the superior and inferior peripapillary area. The improvement of the retinal blood flow ranged from $13.15 \%$ to $16.6 \%$. In 30 normal human volunteers, Kaup et al (2004) found that eyes treated with brinzolamide had no measurable changes in retrobulbar hemodynamic by color Doppler, but did show a significantly reduced arteriovenous passage time compared with placebo, as determined by video fluorescein angiogram.

Theoretically, CAI could give rise to metabolic acidosis, with secondary vasodilatation and improvement of blood flow. Systemic acidosis can occur in the setting of oral CAI therapy, and local acidosis within ocular tissues is theoretically possible with topical CAI therapy, with the potential for a local increase in ocular blood flow (Kaur et al 2002). Topical and systemic CAI have been shown to improve parameters related to ocular blood flow (Harris et al 1996, 1999; Barnes et al 2000). In particular, because systemic CAI also results in altered $\mathrm{CO}_{2}$ clearance and pH levels (Sponsel and Shipman 1997), an increase in tissue $\mathrm{PCO}_{2}$ might promote local vasodilatation. Furthermore, several authors found that acetazolamide is capable of increasing cerebral blood flow in a dose-dependent manner, which was attributed to extracellular acidification (Severinghaus and Cotev 1968; Hauge et al 1983; Volstrup et al 1984; Faraci et al 1987; Ringelstein et al 1992).

Even if local CAIs accumulate inside the red blood cells because of their high affinity for some proteins of red cells and plasma (Harris et al 1996; Costa et al 2003), it has been shown that topical CAI did not affect the retrobulbar hemodynamic 
parameters, while they enhanced the retinal blood flow velocity indices, improved retinal blood flow, and increased the capillary velocity in the superficial vessels of the ONH.

Several studies have shown blood flow or blood velocity differences between normal healthy subjects and glaucomatous ones (Galassi et al 1992; Gherghel et al 2000); and epidemiological studies have disclosed an increased risk for glaucoma when ocular perfusion was low (Tielsch et al 1995; Bonomi et al 2000). Therefore, the secondary vasoactive effect of brinzolamide could be useful in glaucoma since evidence is growing that in some glaucoma patients vascular dysregulation and lower perfusion pressure are important risk factors (Harris et al 1996, 1999; Carlsson et al 2000).

In conclusion, topical CAI treatment has an efficacy in IOP-lowering that ranges from $15 \%$ to $20 \%$. Published data show that brinzolamide can be used as first-line medication, even if other medications have a higher efficacy, with few side effects, and that it is a good adjunctive treatment. In those patients in which IOP must be reduced more than $30 \%$, brinzolamide can be combined with either prostaglandin analogues or with beta-blockers. In the near future new formulations with brinzolamide will be on the market. In particular it will be combined with a beta-blocker or a prostaglandin analogue. In the former fixed combination, both molecules work to decrease IOP by suppressing the production of aqueous humor, but in the latter the target of the two medications to decrease IOP is different. In particular, topical CAI should be able to inhibit the activity of CA in the ciliary epithelium which the prostaglandins tend to increase (Shoji et al 2005; Nakamoto and Franks 2006; Yasuda 2007).

In some types of glaucoma patients with a vascular dysregulation, topical CAI could have a double effect: to reduce the IOP and to improve the ocular blood flow. However, it is still controversial whether topical CAI can improve ocular blood flow, and we have to consider the possibility that the improvement of the ocular flow could be also be due to simply IOP reduction. Even if some clinical studies showed that brinzolamide could improve blood flow, no long-term trial has been performed to answer this question.

\section{Disclosures}

The author has no conflicts of interest to declare.

\section{References}

Adamson I. 1999. Irreversible corneal decompensation in patients treated with topical dorzolamide. Am J Ophthalmol, 128:774-5.

AGIS Investigators. 2000. The Advanced Glaucoma Intervention Study (AGIS): 7. The relationship between control of intraocular pressure and visual field deterioration. Am J Ophthalmol, 130:429-40.
Barnes GE, Wilhelm F, Schoch C. 2000. Increased optic nerve head blood flow after 1 week of twice daily topical brinzolamide treatment in Dutch-belted rabbits. Surv Ophthalmol, 44:S131-S40.

Bonomi L, Marchini G, Maraffa M, et al. 2000. Vascular risk factors for primary open angle glaucoma. Ophthalmology, 107:1287-93.

Budenz DL, Anderson DR, Feuer WJ, et al. 2006. Detection and prognostic significance of optic disc hemorrhages during the Ocular Hypertension Treatment Study. Ophthalmology, 113:2137-43

Carlsson AM, Chauhan BC, Lee AA, et al. 2000. The effect of brimonidine tartrate on retinal blood flow in patients with ocular hypertension. Am J Ophthalmol, 129:297-301.

Collaborative Normal-Tension Glaucoma Study Group. 1998. The effectiveness of intraocular pressure reduction in the treatment of normal-tension glaucoma. Am J Ophthalmol, 126:498-505.

Costa VP, Harris A, Stefansson E, et al. 2003. The effects of antiglaucomatous and systemic medications on ocular blood flow. Progr Retin Eye Res, 22:769-805.

Cvetkvic RS, Perry CM. 2003. Brizolamide. A review of its use in the management of Primary open-angle glaucoma and ocular hypertension. Drug Aging, 20:919-47.

DeSantis L. 2000. Preclinical overview of brinzolamide. Surv Ophthalmol, 44(Suppl 2):S119-S129.

European Glaucoma Society. Terminology and Guidelines 2003. Savona: Dogma.

Faraci FM, Heistad DD, Mayham WG, et al. 1987. Role of larger arteries in regulation of blood flow to the brain stem in cats. $J$ Physiol, 387:112-5.

Feldman RM, Tanna AP, Gross RL, et al. 2007. Comparison of the ocular hypotensive efficacy of adjunctive brimonidine $0.15 \%$ or brinzolamide $1 \%$ in combination with travoprost $0.004 \%$. Ophthalmology, $114: 1248-54$

Franks W. 2006. Ocular hypotensive efficacy and safety of brinzolamide ophthalmic suspension $1 \%$ added to travoprost ophthalmic solution $0.004 \%$ therapy in patients with open-angle glaucoma or ocular hypertension. Curr Med Res Opin, 22:1643-9.

Galassi F, Nuzzaci G, Sodi A, et al. 1992. Color Doppler imaging in evaluation of optic nerve blood supply in normal and glaucomatous subjects. Int Ophthalmol, 16:273-6.

Gherghel D, Orgul S, Gugleta K, et al. 2000. Relation between ocular perfusion and retrobulbar blood flow in patients with glaucoma with progressive damage. Am J Ophthalmol, 130:597-605.

Grieshaber MC, Mozaffarieh M, Flammer J. 2007. What is the link between vascular dysregulation and glaucoma? Surv Ophthalmol, 52(Suppl 2): S144-54.

Harris A, Arend O, Arend S, et al. 1996. Effects of topical dorzolamide on retinal and retrobulbar hemodynamics. Acta Opthalmol Scand, 74:569-72.

Harris A, Arend O, Kagemann L, et al. 1999. Dorzolamide, visual function and ocular hemodynamics in normal tension glaucoma. JOcular Pharm Ther, 15:189-97.

Hauge A, Nicolaysen G, Thoresen M. 1983. Acute effects of acetazolamide on cerebral blood flow in man. Acta Physiol Scand, 117:233-9.

Heijl A, Leske MC, Bengtsson B, et al. 2002. Reduction of intraocular pressure and glaucoma progression: results from the Early Manifest Glaucoma Trial. Arch Ophthalmol, 120:1268-79.

Hollo G, Chiselita D, Petkova N, et al. 2006. The efficacy and safety of timolol maleate versus brinzolamide each given twice daily added to travoprost in patients with ocular hypertension or primary open-angle glaucoma. Eur J Ophthalmol, 16:816-23.

Iester M, Altieri M, Michelson G, et al. 2003. Retinal peripapillary blood flow before and after topical brinzolamide. Ophthalmologica, 218:390-6.

Iester M. 2008. Brinzolamide. Expert Opin Pharmacother, 9:653-62.

Ingram CJ, Brubaker RF. 1999. Effect of brinzolamide and dorzolamide on aqueous humor flow in human eyes. Am J Ophthalmol, 128:292-6.

Kass MA, Heuer DK, Higginbotham EJ, et al. 2002. The Ocular Hypertension Treatment Study: a randomized trial determines that topical ocular hypotensive medication delays or prevents the onset of primary open-angle glaucoma. Arch Ophthalmol, 120:701-13. 
Kaup M, Plange N, Niegel M, et al. 2004. Effects of brinzolamide on ocular haemodynamics in healthy volunteers. Br J Ophthalmol, 88:257-62.

Kaur IP, Smitha R, Deepika A, et al. 2002. Acetazolamide: future perspective in topical glaucoma therapeutics. Int J Pharm, 248:1-14.

Konowal A, Morrison JC, Brown SVL, et al. 1999. Irreversible corneal decompansation in patients treated with topical dorzolamide. Am J ophthalmol, 127:403-6.

Kothy P, Hollo G. 2001. Does glaucoma medication influence the diameter of the retinal arteriole in the human eyes? (A pilot study using the retinal vessel analyser). Aca Phisiol Hungarica, 88:281-92.

Leske MC, Connell AM, Wu SY, et al. 1995. Risk factors for open-angle glaucoma. The Barbados Eye Study. Arch Ophthalmol, 113:918-24.

Leske MC, Heijl A, Hussein M, et al. 2003. Factors for glaucoma progression and the effect of treatment: the early manifest glaucoma trial. Arch Ophthalmol, 121:48-56.

Leske MC, Heijl A, Hyman L, et al. 2007. EMGT Group. Predictors of long-term progression in the early manifest glaucoma trial. Ophthalmology, 114:1965-72.

March WF, Ochsner KI. 2000. The long-term safety and efficacy of brinzolamide $1.0 \%$ (azopt) in patients with primary open-angle glaucoma or ocular hypertension. The Brinzolamide Long-Term Therapy Study Group. Am J Ophthalmol, 129:136-43.

Maren TH. 1967. Carbonic anhydrase: chemistry, physiology, and inhibition. Physiol Rev, 47:595-781.

Martinez-de-la-Casa JM, Castillo A, Garcia-Feijoo J, et al. 2004. Concomitant administration of travoprost and brinzolamide versus fixed latanoprost/timolol combined therapy: three-month comparison of efficacy and safety. Curr Med Res Opin, 20:1333-9.

Menon GJ, Vernon SA. 2006. Topical brinzolamide and metabolic acidosis. Br J Ophthalmol, 90:247-8.

Michaud JE, Friren B. 2001. Comparison of topical brinzolamide 1\% and dorzolamide $2 \%$ eye drops given twice daily in addition to timolol $0.5 \%$ in patients with primary open-angle glaucoma or ocular hypertension. Am J Ophthalmol, 132:235-43.

Nakamoto K, Yasuda N. 2007. Effect of concomitant use of latanoprost and brinzolamide on 24-hour variation of IOP in normal-tension glaucoma. J Glaucoma, 16:352-7.

Nicolela MT, Drance SM. 1996. Various glaucomatous optic nerve appearances: clinical correlations. Ophthalmology, 103:640-9.

Pederson JE, Anderson DR. 1980. The mode of progressive disc cupping in ocular hypertension and glaucoma. Arch Ophthalmol, 98:490-5.

Quigley HA, West SK, Rodriguez J, et al. 2001. The prevalence of glaucoma in a population-based study of Hispanic subjects: Proyecto VER. Arch Ophthalmol, 119:1819-26.

Reis R, Queiroz CF, Santos LC, et al. 2006. A randomized, investigatormasked, 4-week study comparing timolol maleate $0.5 \%$, brinzolamide $1 \%$, and brimonidine tartrate $0.2 \%$ as adjunctive therapies to travoprost $0.004 \%$ in adults with primary open-angle glaucoma or ocular hypertension. Clin Ther, 28:552-9.

Ringelstein EB, Van Eyck S, Mertens I. 1992. Evaluation of cerebral vasomotor reactivity by various vasodilating stimuli: comparison of $\mathrm{CO}_{2}$ to acetazolamide. J Cerebral Blood Flow, 12:162-8.
Sall K. 2000. The efficacy and safety of brinzolamide $1 \%$ ophthalmic suspension (Azopt) as a primary therapy in patients with open-angle glaucoma or ocular hypertension. Brinzolamide Primary Therapy Study Group. Surv Ophthalmol, 44(Suppl 2):S155-S162.

Sampaolesi J, Tosi J, Darchuk V, et al. 2001. Antiglaucomatous drugs effects on optic nerve head flow: design, baseline and preliminary report. Int Ophthalmol, 23:359-67.

Schulzer M, Drance SM. 1987. Intraocular pressure, systemic blood pressure, and age: a correlational study. Br J Ophthalmol, 71:245-9.

Severinghaus JW, Cotev S. 1968. Carbonic acidosis and cerebral vasodilatation after diamox. Scand J Clin Lab Invest, 22:1E.

Shin D. 2000. Adjunctive therapy with brinzolamide 1\% ophthalmic suspension (Azopt) in patients with open-angle glaucoma or ocular hypertension maintained on timolol therapy. Surv Ophthalmol, 44(Suppl 2):S163-S168.

Shoji N, Ogata H, Suyama H, et al. 2005. Intraocular pressure lowering effect of brinzolamide $1.0 \%$ as adjunctive therapy to latanoprost $0.005 \%$ in patients with open angle glaucoma or ocular hypertension: an uncontrolled, open-label study. Curr Med Res Opin, 21:503-8.

Shoji N. 2007. Brinzolamide: efficacy, safety and its role in the management of glaucoma. Expert Rev Ophthalmol, 2:695-704.

Siesky B, Lin T, Kagemann L, et al. 2006. Comparison of three months treatment with dorzolamide and brinzolamide on perfusion pressures in primary open angle glaucoma patients. International Symposium on Ocular Pharmacology and Therapeutics, April, 2006, Berlin.

Silver LH. 1998. Clinical efficacy and safety of brinzolamide (Azopt), a new topical carbonic anhydrase inhibitor for primary open-angle glaucoma and ocular hypertension. Brinzolamide Primary Therapy Study Group. Am J Ophthalmol, 126:400-8.

Silver LH. 2000. Dose-response evaluation of the ocular hypotensive effect of brinzolamide ophthalmic suspension (Azopt). Brinzolamide Dose-Response Study Group. Surv Ophthalmol, 44(Supp1 2): S147-S53.

Sponsel WE, Shipman DL. 1997. The role of blood gases in ocular perfusion. In: Drance S, Chauhan B (eds): Ocular blood flow. Amsterdam: Kugler. Vol 3.

Tielsch JM, Katz J, Sommer A, et al. 1995. Hypertension, perfusion pressure, and primary open-angle glaucoma. A population-based assessment. Arch Ophthalmol, 113:216-21.

Van der Valk R, Webers CAB, Schouten JSAG, et al. 2005. Intraocular pressure-lowering effects of all commonly used glaucoma drugs. A metaanalysis of randomized clinical trials. Ophthalmology, 112:1177-85.

Volstrup S, Henriken L, Paulson DB. 1984. Effect of acetazolamide on cerebral blood flow and cerebral metabolic rate oxigen. J Clin Invest, 74:1634-9.

Wang TH, Huang JY, Hung PT, et al. 2004. Ocular hypotensive effect and safety of brinzolamide ophthalmic solution in open angle glaucoma patients. J Formos Med Assoc, 103:369-73.

Zeyen TG, Caprioli J. 1993. Progression of disc and field damage in early glaucoma. Arch Ophthalmol, 111:62-5.

Zhao JC, Chen T. 2005. Brinzolamide induced reversible corneal decompensation. Br J Ophthalmol, 89:389-90. 
